\title{
Mythes et massacres : reconsidérer la « terreur directoriale »
}

\author{
Howard G. Brown
}

\section{(2) OpenEdition \\ 1 Journals}

Édition électronique

URL : https://journals.openedition.org/ahrf/434

DOI : 10.4000/ahrf.434

ISSN : 1952-403X

\section{Éditeur :}

Armand Colin, Société des études robespierristes

\section{Édition imprimée}

Date de publication : 1 septembre 2001

Pagination : 23-52

ISSN : 0003-4436

\section{Référence électronique}

Howard G. Brown, « Mythes et massacres : reconsidérer la « terreur directoriale » », Annales historiques de la Révolution française [En ligne], 325 | juillet-septembre 2001, mis en ligne le 10 avril 2006, consulté le 24 avril 2022. URL : http://journals.openedition.org/ahrf/434 ; DOI : https://doi.org/10.4000/ahrf. 434

Ce document a été généré automatiquement le 24 avril 2022.

Tous droits réservés 


\title{
Mythes et massacres : reconsidérer la « terreur directoriale »
}

\author{
Howard G. Brown
}

«Que pourroit faire de plus que les cinq directeurs, le tyran le plus farouche et le plus sanguinaire? Robespierre régnoit par la terreur. Ils ont régné par la terreur. Robespierre mutiloit la convention, ils ont mutilé le corps législatif;

Robespierre avoit créé des tribunaux révolutionnaires, ils ont créé des commissions militaires. Robespierre faisoit guillotiner les

émigrés. Ceux-ci les font fusiller. Ainsi s'est r'ouverte sans obstacle et sous une autre forme, l'effroyable boucherie d'hommes que la mort de

Robespierre sembloit avoir irrévocablement

fermée. " ${ }^{1}$

1 À l'aube du 4 septembre 1797, un simple coup de canon retentit dans les rues désertes de Paris. Trente-six heures plus tard, sous la pression de dix-huit mille hommes de troupes dans la région et des preuves accablantes d'un complot anglo-royaliste placardées sur les murs de la capitale, la législature proscrivit deux directeurs, cinquante-trois députés, deux généraux, trois conspirateurs royalistes déjà condamnés et un groupe disparate de cinq suspects. Cependant, pour éviter que de nombreuses décapitations ne souillent la place de la Concorde, récemment rebaptisée, on ordonna la déportation des proscrits. Dix-sept d'entre eux furent arrêtés et déportés en Guyane, dont huit y moururent, donnant lieu au sobriquet de la "guillotine sèche ». La législature avait également annulé les élections du printemps 1797 dans la moitié des départements français, mesure qui entraîna la destitution de cent vingt-deux députés de plus, ainsi que celle de plusieurs centaines d'administrateurs départementaux, de juges et d'accusateurs publics². 
2 Le coup d'État du 18 fructidor anV apporta une riposte vigoureuse à ce qui fut une véritable conspiration royaliste aux ramifications internationales. Cependant, l'ensemble des mesures qu'il entraîna dans sa suite dépassait de loin ce qu'il fallait pour contrecarrer un complot. La soi-disant «terreur directoriale » déclenchée par le coup d'État, ranima la persécution des prêtres réfractaires et des émigrés au nom de la défense de l'ordre républicain. À l'annulation des élections et à la proscription des députés, s'ajoutèrent les articles de la loi du 19 fructidor anV (5 septembre 1797) qui imposèrent à l'ensemble des électeurs, des fonctionnaires et du clergé un serment de haine à la royauté et à l'anarchie, remirent en vigueur les lois tout récemment révoquées de 1792 et 1793 contre les prêtres réfractaires, conférèrent au Directoire le pouvoir d'ordonner des déportations individuelles d'agitateurs ecclésiastiques, interdirent une nouvelle fois aux parents d'émigrés d'exercer des fonctions publiques, et s'efforcèrent de purger la République des émigrés rentrés en France. Les émigrés en détention au moment du coup d'État devaient être déportés; ceux qui tentaient de rester en France ou qui reviendraient plus tard seraient condamnés à mort. Afin de mener à bien cette tâche, la législation rétablit les commissions militaires créées en 1792 dont le seul objet était de décider si le particulier comparu devant elles correspondait bien au nom inscrit sur la liste officielle d'émigrés. Dans ce cas, l'accusé devait être exécuté dans les vingt-quatre heures. En cas de désaccord, les autorités civiles devaient trancher ${ }^{3}$. Ces mesures, selon Jean-Pierre Gallois, rouvrirent "l'effroyable boucherie d'hommes que la mort de Robespierre sembloit avoir irrévocablement fermée ». C'est ainsi que les commissions militaires fructidoriennes devinrent l'instrument définitif de la «terreur directoriale ».

3 Le parallélisme entre la répression de 1793-1794 et celle de 1797-1799, noté explicitement par Gallois et même par Toqueville, qui affirma que certaines lois du Directoire étaient plus barbares que celles de la Terreur ${ }^{4}$, est fait implicitement par les historiens récents. Presque toutes les études historiques consacrées à la période du Directoire font référence soit à la «terreur directoriale» soit à la «terreur fructidorienne $»^{5}$. Le concept de «terreur directoriale» doit beaucoup à l'œuvre de Victor Pierre. Ses trois livres offrent une vaste documentation sur les commissions militaires du soi-disant Second Directoire et ses déportations d'ecclésiastiques ${ }^{6}$. Ces travaux n'ont pas été révisés depuis un siècle et servent toujours de référence à l'ensemble des travaux historiques de cette période. Les recherches de Victor Pierre sur les commissions militaires fructidoriennes, apparemment exhaustives, lui avaient permis d'identifier deux cents prévenus parmi lesquels cent quatre avaient été exécutés. À ce nombre Pierre ajouta, grâce à un relevé de facture établi pour l'excavation de fosses à Toulon, cinquante-quatre autres personnes non identifiées ${ }^{7}$. Croyant qu'il y en avait plusieurs qui lui manquaient, il arrondit le résultat à cent soixante exécutions. C'est ce chiffre qui apparaît dans presque toutes les études de synthèse publiées au vingtième siècle ${ }^{8}$ et depuis, les historiens ont toujours ajouté foi aux conclusions qu'il avait tirées de sa documentation. D'après lui, à part quelques femmes et un total de quarante et un ecclésiastiques "déportés ", exécutés en tant qu'émigrés revenus d'exil, «le surplus des victimes (108) se compose de prévenus d'émigration à qui, en grande majorité, on ne pouvait reprocher que d'être rentrés en France ou de n'en être pas sortis à temps $»^{9}$. Il en conclut, par ailleurs, que ces commissions, créées par "une législation perfide et barbare", prirent fin lors de la retraite forcée de Treilhard, Merlin et La Révellière-Lépaux du Directoire, le 30prairial anVII (18 juin 1799). Même les quelques historiens scrupuleux qui décident de passer au 
crible la documentation réunie par Victor Pierre, sont obligés d'en conclure que les commissions déclaraient coupables sept personnes sur dix, que les trois quarts des condamnés étaient des ecclésiastiques et d'anciens nobles et que la légalité des acquittements était souvent douteuse ${ }^{10}$. Il s'agissait donc d'une persécution brutale, anti-cléricale, anti-nobiliaire et maladroite.

Malgré l'effort extraordinaire consacré par Victor Pierre à sa tâche, il restait un point faible dans sa documentation. Victor Pierre s'était en effet persuadé que les archives militaires ne contenaient aucune documentation sur le travail de ces commissions et que le ministère de la Guerre en ignorait absolument les activités. Or, les recherches que nous avons menées au Service historique de l'Armée de Terre et dans les dépôts de province nous autorisent à dire qu'il n'en est rien. À la lumière de plus de mille jugements que nous sommes parvenus à localiser, dont la plupart se trouvent à Vincennes ${ }^{11}$, une révision s'avère nécessaire. Bien qu'il soit impossible d'établir une liste définitive des jugements, cette profusion de nouvelles preuves modifie certains aspects fondamentaux de la terreur directoriale. Les commissions militaires siégèrent dans 50 cités et villes différentes et rendirent 1006 jugements à l'encontre de 975 individus ${ }^{12}$. Ces jugements comprenaient la condamnation de 274 individus à mort, 93 à la déportation, 24 à l'exil et 22 à des peines d'emprisonnement (voir tableau infra p. 44). Ceci constitue une augmentation significative du nombre des exécutions et expulsions par rapport à ce qui était connu précédemment, mais au total ce nombre ne représente un taux de condamnation que de 4 sur 10 seulement, beaucoup plus bas que le taux de Victor Pierre de presque 7 sur 10. Afin d'expliquer ce fait paradoxal d'un plus grand nombre de victimes mais d'un taux de condamnation inférieur, il est nécessaire de reconsidérer la terreur directoriale et plus particulièrement le rôle que les commissions militaires y ont joué.

Un examen de la terreur directoriale doit commencer par l'étude de ses origines en amont du coup d'État du 18 fructidor. Le républicanisme du Second Directoire ne peut être compris qu'en le considérant comme un ensemble de pratiques discursives et de réflexes conditionnés. Ce genre de politique s'est forgé aux moments forts de la jeune République, entre 1792 et 1795 , sur un fond de violence, de répression et de guerre à outrance. Ainsi, cette forme de républicanisme fut à la fois solide et peu flexible, le produit d'une praxis révolutionnaire ${ }^{13}$. Au cours de l'année suivant la chute de Robespierre, des républicains de toutes sortes avaient à confronter les problèmes fondamentaux liés à leur expérience révolutionnaire. Dans un premier temps, il leur fallait trouver un moyen de sortir de la Terreur, ensuite de sortir de la Révolution elle-même. Forts de leurs épreuves, les conventionnels thermidoriens avaient répudié la démocratie radicale de l'anII. À l'automne 1795, ils avaient restreint le pouvoir aux mains des propriétaires, mis un barrage constitutionnel entre le pouvoir politique et la liberté individuelle et imposé une application impartiale de la loi comme une des notions clés de la sécurité personnelle. On ne peut nier la volonté exprimée par ces hommes de tourner la page, de rétablir un ordre politique et une société civile pacifiés, réconciliés sous le règne de la $\operatorname{loi}^{14}$. Mais il est vrai aussi que la république constitutionnelle s'était compromise dès sa naissance. La loi des Deux-tiers votée par la Convention finissante restreignait le choix de l'électorat déjà en 1795, l'amnistie des crimes politiques ne s'appliquait pas aux insurgés des 13-14 vendémiaireanIV (15-16 octobre 1795), les parents d'émigrés étaient déclarés inaptes à remplir des fonctions publiques et la Constitution de l'an III interdisait formellement toute nouvelle loi permettant le retour des émigrés. Ce renouvellement des persécutions contre les 
prêtres et les émigrés risquait de miner la légitimité démocratique et républicaine d'un régime reposant sur les fondements constitutionnels de la séparation des pouvoirs judiciaire et exécutif et sur les bases morales d'une Déclaration des droits de l'homme.

6 Pendant les deux premières années du Directoire, le débat politique fit apparaître souvent des tensions inhérentes au libéralisme démocratique -bien que les arguments pour la défense de la Constitution, de la liberté de la presse, de la citoyenneté et du droit de vote, fussent souvent motivés autant par des considérations de lutte entre les factions que par des considérations de principe. Le débat politique s'articulait aussi autour de discours concurrentiels d'une constitutionnalité rigide et d'un régime révolutionnaire d'exception. Le sujet le plus controversé du débat était la loi du 3 brumaire anIV (22 octobre 1795) qui remit en vigueur les lois anticléricales de 1792-1793 et qui excluait les proches parents d'émigrés des fonctions publiques. Naturellement, les conservateurs voyaient dans les prêtres réfractaires et les parents d'émigrés des alliés potentiels et firent usage du langage de la liberté afin de discréditer la politique d'exclusion. À la défense de cette législation, les républicains ardents la qualifiaient de rempart essentiel contre une restauration de la monarchie et en fait ils réussirent à la préserver presque intégralement jusqu'au triomphe de la droite aux élections du printemps $1797^{15}$. C'est alors que, dans l'espace de trois mois, les législateurs permirent aux prêtres réfractaires et aux parents d'émigrés revenus d'exil de rejoindre le corps politique, tentant ainsi un retour au libéralisme politique du début de la Révolution. On vit soudain une foule de prêtres réfractaires et d'émigrés rentrer en France, ou surgir des cabanes forestières et des fermes isolées où ils s'étaient réfugiés. On put constater la cessation de la vente des biens nationaux et l'augmentation notable du crime et des désordres. Le pays subit de nombreux incidents de violence politique, tels l'assassinat près de Bordeaux de Groussac, maire de Toulouse en l'anII, le siège et le massacre des membres du présumé Cercle constitutionnel de Clermont-Ferrand et l'escalade des heurts entre les "ganses blanches » et les « ganses jaunes» dans les environs de Castres. Dans chacun des cas, les magistrats locaux se montrèrent peu disposés à engager des poursuites ${ }^{16}$. À Paris, on croyait assister à l'enfoncement de l'autorité républicaine dans le bourbier de l'attentisme ${ }^{17}$.

7 Les événements de l'été de 1797 avaient servi à confirmer les pires craintes des républicains à l'égard des origines et de l'ardeur du sentiment antirépublicain. À l'époque, on justifiait le coup d'État de fructidor par la nécessité de contrecarrer un complot royaliste, mais son but réel était de rétablir l'autorité du gouvernement. C'est l'effondrement général de l'ordre public pendant l'été de 1797, ainsi que la perception qu'avaient les républicains de leur expérience révolutionnaire qui déterminèrent le cours des actions entreprises. Car la plupart des républicains directoriaux n'adhéraient pas à la thèse de Benjamin Constant ${ }^{18}$ selon laquelle la République avait été sauvegardée malgré la Terreur et qu'il suffisait de persuader les modérés que tout soutien apporté aux réactionnaires ne ferait qu'encourager davantage l'instabilité. À l'encontre de cette proposition lénifiante et rassembleuse, les républicains engagés ne cessaient d'insister, dans leurs discours, sur les menaces multiples, graves et répétées qui pesaient sur la Révolution et auxquelles il fallait répondre par une vigilance constante et une vigueur aggressive ${ }^{19}$. Ils restaient trop conditionnés par leur passé révolutionnaire pour renoncer totalement aux réflexes autoritaires face aux menaces qui pesaient sur la jeune et fragile république. 
8 En dépit de ces raisons invoquées pour un retour à des mesures exceptionnelles, l'inquiétude produite par les entorses données à la légitimité constitutionnelle par la loi du 19 fructidor anV força le gouvernement à en modifier ses applications. L'atténuation de la loi la rendit moins idéologique au prix d'une revalorisation de ses aspects autoritaires et dictatoriaux. Au commencement, les commissions militaires étaient des instruments de terreur rudimentaires et leurs victimes comprenaient inévitablement de pauvres individus qui n'avaient rien fait de pire que de passer outre aux lois d'émigration. La survie de la République dépendait-elle vraiment du passage de trois veuves devant les pelotons d'exécution à Toulon et à Marseille? Quel degré d'insécurité fallait-il pour fusiller un cultivateur âgé de 68 ans à Douai, un autre âgé de 71 ans à Avignon, un prêtre âgé de 76 ans à Marseille et pour déporter un garçon de Mézières de 14 ans $^{20}$ ? Même si en fait ces cas restent exceptionnels, le Directoire s'embarrassa de l'arbitraire des commissions et d'injustices grossières. Il n'était pas disposé à en accepter ces excès.

9 Afin de diminuer de telles cruautés inutiles, aussi bien que de concentrer les commissions sur les véritables ennemis de la République, le Directoire désigna des catégories d'exemptions. Cela réduisit considérablement le nombre d'émigrés susceptibles de passer devant les commissions militaires ${ }^{21}$. Ces exemptions furent créées par des arrêtés ministériels émis sans tambour ni trompette, ni explication; cependant, nous pouvons discerner la raison du choix de chaque catégorie en analysant le débat politique qui régnait avant Fructidor. Par la suite, on peut démontrer l'effet cumulé de ces exemptions en examinant les opérations des commissions militaires.

10 La première exemption du Directoire fut appliquée à des hommes désignés depuis longtemps déjà comme ennemis de la République: les prêtres. En abrogeant les lois contre les prêtres réfractaires peu avant le coup d'État, les Conseils avaient inversé une politique pourtant au cœur du républicanisme dès ses origines. Inévitablement, la loi du 19 fructidor anV devait rétablir cette discrimination. D'abord, elle assimila à nouveau les prêtres réfractaires aux émigrés et les soumit à la peine de mort $^{22}$, avec comme seule différence le fait qu'au lieu de bénéficier de l'immunité de fait accordée par la justice civile, ils risquaient maintenant la rigueur des commissions militaires. Toutefois, le gouvernement s'empressa de rétablir les distinctions datant de 1792, atténuant ainsi la répression ${ }^{23}$ : tous les réfractaires âgés et infirmes, sans tenir compte de leurs différentes pérégrinations, devaient être internés sous surveillance administrative et les prêtres déportés, mais rentrés en France, devaient être expulsés de nouveau. On ne renvoya devant le peloton d'exécution que ceux partis «volontairement» en exil. Néanmoins, les prêtres rétractaires âgés de moins de soixante ans et en assez bonne santé pour voyager, risquaient à nouveau la peine de mort en cas d'arrestation, soit qu'ils eussent quitté la France soit qu'ils se soient simplement tenus discrètement à l'écart pendant les cinq dernières années. Cependant, même cette provision sembla s'atténuer, du moins en pratique. Après les premiers mois, la commission militaire de Besançon refusa de considérer les prêtres exilés comme émigrés; celle de Bayonne exprima une inquiétude bien justifiée devant des listes peu soignées et peu précises des émigrés et renvoya ainsi tous les prêtres devant les autorités civiles ${ }^{24}$.

11 La deuxième sorte d'exemption fut appliquée en fonction de la date politique de l'émigration. Elle était étroitement déterminée par le groupe social auquel appartenait l'émigré en question. Quatre mois après la loi du 19 fructidor, le Directoire exempta les 
ouvriers et les paysans qui avaient fui la France après la prise du pouvoir par les Montagnards en juin 1793 et qui avaient enregistré leur retour en l'an III $^{25}$. Ainsi le Directoire avait choisi de suivre la version de la Terreur inventée au cours des débats thermidoriens sur les lois de l'émigration. À ce moment-là, on discutait pour savoir s'il y avait une différence réelle entre émigrés et fugitifs pour finir par définir les émigrés comme étant ceux qui avaient déserté la patrie pendant sa période de régénération; par contre ceux qui avaient fui les représailles terroristes n'étaient que des fugitifs ${ }^{26}$. Le Second Directoire exempta donc une grande partie des gens des classes laborieuses qui avaient quitté la France après le 31 mai 1793, en particulier les Alsaciens et les Toulonnais qui avaient fui devant la répression menée par les représentants en mission montagnards ${ }^{27}$. Mais celui qui avait quitté la France avant le 31 mai 1793 ne reçut aucune considération spéciale, étant considéré, comme l'énonçait le député Riou, comme «un véritable émigré, c'est-à-dire, l'ennemi le plus dangereux et le plus irréconciliable de la République ${ }^{28}$.

Le Directoire en vint à formuler une troisième sorte d'exemption, qui devait s'appliquer même aux véritables émigrés: celle du sexe. Après la condamnation à mort de quatre femmes (on en exécuta trois et on accorda un sursis à la quatrième pour cause de grossesse), le ministre de la Police générale ordonna de suspendre la condamnation des femmes émigrées et de renvoyer chaque cas de ce genre au gouvernement ${ }^{29}$. Bien que les commissions militaires eussent déjà exilé seize femmes, la directive du ministre mit fin à presque toutes les poursuites. Le petit nombre de femmes apparaissant ultérieurement devant les commissions militaires furent soit acquittées soit exemptées ${ }^{30}$.

La quatrième sorte d'exemption visait l'appartenance sociale des émigrés. En juillet 1798, l'exemption politique accordée aux classes laborieuses fut étendue à tous les ouvriers, artisans et paysans, même s'ils n'avaient pas rempli les conditions de la législation thermidorienne ${ }^{31}$, redéfinition qu'on avait failli adopter déjà une année auparavant au cours d'un débat législatif envenimé. Cependant, le gouvernement avait toujours refusé d'exempter les négociants ou les commerçants ${ }^{32}$. Ce refus les plaçait dans la même catégorie que les anciens nobles et les prêtres "déportés ». Il en fut de même pour ceux qui vivaient de leurs biens ou de leurs professions. Tous étaient, du fait même de leurs professions, justiciables d'une commission militaire. Cette inclusion explicite était, en apparence, une absurdité et les conservateurs n'avait pas manqué de fustiger de tels préjugés fondés sur l'appartenance sociale. Pendant le débat sur les réfugiés, vers la fin de l'an III, Tronson-Ducoudray avait soutenu avec force que de telles lois faisaient fi de la responsabilité personnelle parce qu'elles laissaient entendre qu'» un citoyen est innocent parce qu'il appartient à telle classe; [qu'il] est coupable parce qu'il appartient à telle autre: ainsi le propriétaire, le négociant, l'homme de lettres, l'homme de loi, le rentier sont criminels... il y a [une] caste privilégiée pour l'échafaud $»^{33}$. Et pourtant, poursuivait-il, ceux qui avait donné Toulon aux Anglais, avaient été réduits à cette mesure désespérée par la logique d'une répression de plus en plus acharnée après le 2 juin 1793; en conséquence, ne devrait-on pas absoudre les auteurs de la trahison collective envers la République naissante de toute responsabilité individuelle? Démonstration brillante, mais qui profita peu soit aux émigrés de 1793 soit à son auteur: Tronson-Ducoudray devait bientôt mourir en Guyane, victime à son tour de la terreur fructidorienne. 
14 Ainsi, seulement quelques mois après la loi du 19 fructidor, le gouvernement avait, par ses différents arrêtés, exempté partiellement ou complètement la plupart des émigrés des rigueurs d'une commission militaire. Absurdité ? Facilité ? Insouciance ? Non. Cette politique d'exemption constitue en fait la clé pour comprendre la terreur fructidorienne et, en particulier, le travail des commissions militaires. Les catégories d'exemptions ou, autrement dit, les groupes vraiment visés, avaient été créés par la politique d'auto-défense républicaine et fonctionnèrent en conséquence. Le concept de groupes cibles fut d'abord formulé par la défiance, sinon l'opposition pure et simple, que ces catégories sociales avaient généralement montrée à l'égard des régimes révolutionnaires, y compris évidemment la Terreur. Ce concept de groupe cible fut ensuite perfectionné dans le cadre de la crise du Directoire, la lassitude et le désordre public aidant. Les commissions militaires n'étaient pas prévues pour les émigrés en tant que tels, mais pour des hommes que leur statut socio-économique dotait d'influence et que le Directoire jugeait coupables de mépriser la loi dans le seul but de fomenter une opposition à la République. Aux yeux du Directoire, ces personnes représentaient une menace pour la stabilité sociale et politique aussi importante que celle causée par des voleurs de grand chemin. Bref, le gouvernement considérait le travail des commissions militaires comme faisant partie d'un ensemble de mesures exceptionnelles adoptées pour rétablir l'ordre et consolider une république libérale.

15 Par ironie du sort, c'est pendant une période où on s'efforçait de garantir davantage les libertés qu'on a voulu faire fonctionner cet appareil répressif. C'est que la défense de la liberté personnelle des citoyens avait bien besoin d'un rajout d'efficacité. Chaque jour, le gouvernement ne fut-il pas assailli par une litanie de rapports contenant des détails effrayants de courriers interceptés, d'attaques de diligences, d'assassinats de fonctionnaires, d'intimidation de témoins. L'horrifiante prolifération des " chauffeurs ", en particulier, poussa le législateur à condamner à mort, par la loi du 15 mai 1797, les voleurs armés qui avaient battu ou torturé leurs victimes. Après le coup d'État de Fructidor, le Directoire employa des moyens militaires de répression afin de mettre un terme à «la pire vague de criminalité dans la France des temps contemporains $»^{34}$. La loi draconienne du 18 janvier 1798 définissait les vols avec effraction de résidence et les vols à main armée comme crimes capitaux, justiciables de procédures expéditives devant les tribunaux militaires, s'ils avaient été commis par plus de deux personnes. Pendant les deux années au cours desquelles cette loi fut en usage, les conseils de guerre condamnèrent et exécutèrent plusieurs centaines de civils pour actes de brigandage et de résistance armée contre l'autorité. Le Directoire combina cette justice expéditive avec d'autres mesures de répression telles que la mise en état de siège de plus de deux cents communes et l'imposition de fortes amendes aux communautés qui n'avaient pas pu prévenir les crimes collectifs commis sur leurs territoires. En même temps, on renforça les compagnies de la garde nationale de troupes régulières afin de former des colonnes mobiles pour surveiller routes et campagnes ${ }^{35}$.

16 Car le recours aux commissions militaires pour poursuivre les émigrés en justice s'harmonisait très bien avec les autres méthodes autoritaires et militaires mises en place afin de rétablir l'ordre. Ce fait a été masqué par le traitement historique des commissions militaires uniquement comme instrument de la "terreur directoriale", réduisant ainsi leur tâche répressive à une simple opération politique. En examinant le genre de personnes qui furent jugées par les commissions militaires, on se rend compte 
aisément comment, sous le Second Directoire, les problèmes relevant de l'ordre public et de la stabilité politique se conjuguèrent. Le plus étonnant, lorsqu'on connaît la thèse de Victor Pierre, est que moins d'un quart des individus traduits en justice par les commissions militaires étaient des prêtres ou des nobles. En outre, même en tenant compte de l'exemption des émigrés appartenant aux classes laborieuses, les nobles et les prêtres ensemble constituaient seulement un tiers des prévenus reconnus coupables d'émigration. Malgré l'impression laissée par toute l'historiographie de la terreur fructidorienne, le fait est que la plupart des prisonniers avaient été condamnés pour ce qu'ils avaient fait, non pas seulement pour ce qu'ils étaient. C'est pour cette raison et non pas à cause d'une culpabilité collective présumée, que des prêtres et des nobles furent individuellement jugés et condamnés comme ennemis déclarés de la République.

17 Le Directoire ne faisait aucune confiance aux prêtres en général, bien sûr, mais il s'acharnait particulièrement contre les prêtres réfractaires hardis et itinérants, rendus responsables de l'hostilité populaire envers la République. Les républicains attribuaient, avant tout, la Vendée militaire et la chouannerie au fanatisme des prêtres. En septembre 1797, les républicains croyaient ce mouvement subversif en train de gagner toute la France. À leurs yeux, cette thèse, que nous pourrions croire simpliste, ne manquait pas de preuves. Ils pouvaient citer l'exemple de Joseph Poirot, un prêtre réfractaire des Vosges de retour d'exil en novembre 1796, arrêté pour distribution de pamphlets incendiaires dénonçant le Directoire, condamné à mort et fusillé sans tarder par la commission militaire de Nancy ${ }^{36}$. Cette période était aussi l'âge d'or des prêtresbrigands. Jean-Baptiste Robert, ex-curé, était le chef d'une bande de brigands dans la Lozère. Il n'avait réussi à s'évader de la citadelle de Mîmes deux heures avant d'être fusillé, que pour être repris et exécuté à Montpellier une année plus $\operatorname{tard}^{37}$. Le sort du vicaire Jean-Joseph Glatier fut réglé plus difficilement. Arrêté les armes à la main, en possession de biens acquis lors d'un vol de grand chemin et accompagné de trois gardes du corps chouans, Glatier admit volontiers avoir prêché le retour de la monarchie lors de ses messes clandestines. Il fut dûment condamné et exécuté à Tours ${ }^{38}$. Il est vrai que la culpabilité trop évidente de ces hommes laissait peu de choix aux tribunaux. La plupart de leurs collègues, prêtres insermentés de toutes sortes, qui parurent devant les commissions militaires, furent l'objet d'une clémence surprenante - surtout si l'on considère leur réputation sanguinaire. Certes, la croyance persistante des républicains à une conspiration catholique entraîna la condamnation et l'exécution de quarantehuit ecclésiastiques. Mais, malgré la condamnation de vingt-deux autres à la déportation, seulement six d'entre eux eurent à subir ce sort. On se contenta de la simple expulsion du sol français des quatorze autres prévenus, ou de leur internement dans des maisons d'arrêt, en les assimilant aux dix prêtres condamnés à réclusion dû à leur âge ou à leur santé défaillante.

Le Directoire n'accordait pas davantage de confiance aux ci-devant nobles qu'aux prêtres. Il évita soigneusement de laisser exclure les nobles émigrés de toute exemption à la loi du 19 fructidor. Cette discrimination naquit de la perspective républicaine exprimée lors des débats qui suivirent Fructidor, concernant l'exclusion des nobles de leurs droits civiques. Dix semaines après Fructidor, les conseils législatifs votèrent la loi du 9 frimaire anVI $(29$ novembre 1797) fermant l'arène politique aux ci-devant nobles à moins de pouvoir démontrer des services rendus à la Révolution. C'est alors que Crassous, député au Conseil des Cinq-Cents, sembla atteindre un sommet d'intolérance en soutenant que les nobles avaient démontré leur trahison en abusant de leurs droits politiques - c'est-à-dire en soutenant les conservateurs et les royalistes aux élections - 
et, que par conséquent, ces droits devraient être suspendus provisoirement afin de "préserver le pacte social " $^{39}$. Le député directorial Creuzé-Latouche adopta une optique plus large. Il soutenait que l'expérience révolutionnaire avait démontré jusqu'à présent que la République avait plus à craindre de «la classe des richesses » que de celle des nobles seuls. En fait, il maintenait que la seule noblesse dont la République devait se méfier - sans l'opprimer - était celle de la richesse ${ }^{40}$. Ses collègues le huèrent. Néanmoins, ce fut là précisément la politique du gouvernement envers les notables émigrés.

Il est nécessaire de souligner la différence entre la persécution des nobles et des prêtres en tant que tels et la limitation des commissions militaires à l'engagement de poursuites contre des notables émigrés. Patrice Higonnet a voulu voir dans la loi du 9 frimaire an VI l'apogée de l'idéologie antinobiliaire de la période révolutionnaire, tout en admettant qu'elle eut très peu d'effet à un niveau pratique parce qu'on ne la dota jamais de la législation nécessaire à sa mise en vigueur ${ }^{41}$. Ce manque d'efficacité antinobiliaire aurait toutefois été compensé, toujours selon Higonnet, par la persécution menée par les commissions militaires, qui aurait visé en premier lieu la ci-devant noblesse. Autrement dit, c'était un retour supposé à la formule «émigré = noble » de 1791-1792. Dans cette optique, les commissions militaires créées par la loi du 19 fructidor anV seraient le sous-produit d'une idéologie révolutionnaire plus ancienne élaborée bien avant le passage au régime républicain.

Il ne fait aucun doute que le Directoire prenait parti contre les nobles et que les commissions militaires ont bien répercuté cette politique. Dépeindre cependant l'action de ces commissions comme une simple persécution idéologique aboutirait à effacer, à tort, leurs origines dans l'auto-défense républicaine. En outre, une approche fixée sur l'idéologie politique ignore l'impératif, bien ressenti par le Directoire, de rétablir l'ordre dans un pays tourmenté par la violence. Ce n'est pas une idéologie antinobiliaire élaborée en 1789-1791 qui détermina le choix des émigrés à persécuter.

Le Directoire s'acharna contre les individus ayant commis un acte politiquement surchargé - l'acte de quitter la France révolutionnaire - et même là, il ne considéra pas tous les émigrés comme ennemis. Il chercha plutôt à cerner le sous-groupe des émigrés présentant un danger pour la stabilité de la République. Le gouvernement utilisa donc les commissions militaires principalement pour persécuter les notables émigrés, non pas les ci-devant nobles en tant que tels, ni même tous les émigrés en tant que tels.

Comme les catégories d'exemption accordées aux émigrés l'indiquent, plus que des nobles ou des prêtres en soi, le Directoire s'inquiétait particulièrement de la présence d'émigrés possédant un statut social suffisamment élevé pour leur conférer une certaine influence. Le travail des commissions militaires témoigne amplement de cette inquiétude. La proportion des nobles et des prêtres parmi les accusés exécutés était à peu de chose près identique - $16 \%$ et $18 \%$ respectivement - mais la proportion des condamnés à mort qualifiés de notables, sans être ecclésiastiques ni nobles, est du même ordre (17 \%) (voir tableau infra pp. 45-52). Ces chiffres nous forcent à reconnaître que le Directoire avait visé, par les commissions militaires, l'ensemble des notables émigrés et non pas uniquement les nobles et les prêtres. C'est seulement en comptant ensemble les négociants, les bourgeois, les rentiers, les chirurgiens, les avocats, les artistes, les sous-officiers, les ecclésiastiques et les nobles, que l'on arrive à la moitié du nombre des personnes exécutées par les commissions militaires. L'autre moitié appartenait aux classes laborieuses ${ }^{42}$. Ce grand éventail de victimes indique que la 
responsabilité individuelle joua un rôle bien plus important que ne l'avait anticipé Ducoudray-Tronson dans le sort réservé aux prévenus.

Ces données rendent évident le fait que les commissions militaires s'occupaient en particulier d'hommes présentant un sérieux danger pour la sécurité collective sinon des Français, du moins des républicains. Les archives des commissions sont pleines de cas de notables de tous les états, ayant quitté la France afin de prendre les armes contre la République. La commission militaire de Nice exécuta trois Niçois pour avoir servi comme officiers dans l'armée de Sardaigne ${ }^{43}$. Parmi les condamnés à mort pour avoir servi dans l'armée de Condé figuraient Victor Cazeneuve, rentier de Paris, Jean-Pierre Guy de Villeneuve, ancien officier de cavalerie de Belfort et Philibert, chevalier de la Bussière, un ci-devant seigneur en provenance du Poitou ${ }^{44}$. Le comte de Rochecotte fut peut-être la victime la plus célèbre des commissions militaires fructidoriennes. Il s'était distingué dans l'armée des princes avant de devenir commandant de division dans l'« armée catholique et royale du Maine ». Son arrestation à Paris avait coûté la vie à deux hommes ${ }^{45}$. Une autre victime aristocratique, le marquis de Surville, l'un des principaux agents du Prétendant dans le Midi, organisait des opérations contrerévolutionnaires, qu'il subventionnait par des attaques contre des diligences et au moyen de faux monnayage ${ }^{46}$. Il ne s'agissait pas simplement de cas isolés d'opérations de guérilla ou de crimes orchestrés par des émigrés. Quarante-sept officiers de l'« armée catholique et royale de Normandie » étaient des émigrés rentrés en France ${ }^{47}$. Il est donc peu surprenant que le comte de Frotté et ses compagnons furent jugés à la hâte et exécutés par une commission militaire en pluviôse an VIII, sans considération aucune pour leur possession d'un sauf-conduit ni pour l'absence de constats d'émigration requis par la loi du 19 fructidor.

Les commissions militaires exécutaient aussi des émigrés sans se soucier de leur statut social, s'ils avaient commis antérieurement des crimes contre la République. Par exemple, les commissions militaires de Toulon et de Marseille, qui comptaient parmi les plus actives, ${ }^{48}$ ignoraient l'amnistie du 4 brumaire an IV (26 octobre 1795) concernant les actes liés à la Révolution et appliquaient les lois pourtant abrogées de l'an III, afin de distinguer les fugitifs toulonnais des émigrés qui avaient collaboré avec les Anglais. C'est ainsi que des douzaines de prévenus qui avaient fui la France pendant l'été et l'automne 1793 s'étaient crus, à tort, à l'abri de la punition en obéissant à la loi, qui leur prescrivait de se présenter auprès de leurs municipalités. Une vingtaine d'autres, qui dans des circonstances différentes auraient bénéficié d'une exemption furent exécutés, certains pour avoir fourni des provisions, d'autres pour avoir servi comme gendarmes, un pour sa participation à l'incendie de la flotte française et un autre pour avoir servi dans une cour martiale dont les jugements étaient datés de «l'an premier du règne de Louis XVII $»^{49}$. De la même manière, des brigands émigrés au statut social très varié, par exemple le chevalier Gérard de Saint-Elme, le laboureur Antoine Laquerre dit l'Intrépide et Pierre-Charles Yvon, sans profession, finirent leurs jours à la suite de leur audition par des commissions militaires ${ }^{50}$. Ces histoires individuelles montrent clairement que beaucoup de victimes des commissions militaires étaient des hommes dont le comportement et l'attitude constituaient un jour ou l'autre une menace sérieuse pour la stabilité du régime. Le Directoire s'embarrassa peu de la politique menée contre de telles victimes malgré les violations des lois et parut disposé à en accepter les conséquences. En outre, l'arbitraire des commissions paraît bien peu 
de chose en comparaison des actes bien arbitraires commis par le gouvernement luimême envers les prêtres.

Car la «terreur directoriale» impliqua davantage que l'existence de commissions militaires. La loi du 19 fructidor non seulement envoyait les députés et les conspirateurs royalistes à la "guillotine sèche ", elle autorisait le Directoire à émettre de son propre chef des arrêtés pour la déportation individuelle des prêtres, sans procès ni instruction judiciaire. En cette matière, le Directoire abusa énormément de son pouvoir et il autorisa les administrateurs départementaux à l'imiter. Par conséquent, des ordres purement administratifs entraînèrent la déportation de près de 1400 prêtres, dont 187 moururent dans l'espace de deux ans ${ }^{51}$. C'est ainsi que trois fois plus de prêtres sont morts en déportation que par exécution.

Bien que les commissions militaires aient également condamné quatre-vingt-treize personnes à la "déportation ", le nombre réel de déportés était en réalité infime seulement cinq prisonniers furent envoyés en Guyane [trois y moururent ${ }^{52}$ ] et treize aux îles de Ré et d'Oléron. De ceux qui restaient, le plus grand nombre fut simplement expulsé de France, y compris quarante-six personnes jugées à Strasbourg qui, dans ce cas, connurent le même sort que les vingt-six personnes condamnées à la "réémigration" par les commissions de Marseille et de Toulon ${ }^{53}$. Les commissions militaires eurent recours à l'expulsion dans les cas de personnes déclarées incapables d'obéir à la loi du 19 fructidor pour cause d'emprisonnement, de maladie ou d'infirmité (y compris l'aliénation mentale et la vieillesse) et lorsque les émigrés avaient été arrêtés sur un territoire envahi par l'armée française. Un certain nombre d'émigrés furent aussi expulsés illégalement. Cette arme dans l'arsenal des autorités pouvait être à double tranchant comme l'illustrent deux cas jugés à Saint-Brieuc. Sébastien Guillemain, un prêtre réfractaire de 66ans, avait été condamné par contumace à la « réclusion" par un tribunal criminel ordinaire. Cependant, arrêté après Fructidor, il fut condamné par la commission militaire de Saint-Brieuc à la déportation. Tout en admettant que Guillemain n'avait pas fui la France, ses membres prétendaient «que dans le moment où des hordes de brigands armés infestent le département des Côtesdu-Nord, la présence dudit Guillemain sur le territoire de la République ne peut être qu'infiniment dangereuse au maintien de l'ordre »- une évidente transgression de la loi et une usurpation du pouvoir des autorités civiles. D'autre part, Hyacinthe-Marie Crésoler-Trélin, ancien noble et officier de marine, eut sa vie épargnée lorsque quatre membres votèrent pour la déportation, mettant ainsi en minorité les trois qui se prononcèrent (comme ils le devaient) pour la peine de mort ${ }^{54}$. D'autres commissions furent coupables d'autres irrégularités telles que d'avoir prononcé une douzaine de peines de prison cumulées à l'encontre de complices d'émigrés, de prisonniers de guerre évadés et de personnes qui avaient émigré bien avant la Révolution ${ }^{55}$. En outre, l'apparente clémence des tribunaux provoqua parfois une épidémie de dénonciations de la part des autorités départementales intransigeantes, ce qui poussa le Directoire à annuler deux douzaines d'acquittements, quitte à durcir certains jugements trop cléments lorsque les commissions militaires avaient outrepassé leur autoritéé ${ }^{56}$.

Les inscriptions sur la liste des émigrés, les attestations de résidence et les certificats de maladie faisaient tous l'objet d'un trafic considérable d'argent et d'influence; le gouvernement devait ainsi faire preuve d'une vigilance constante afin de s'assurer que seules les administrations départementales et non pas les commissions militaires, vérifiaient l'authenticité de ces documents. La dépendance des commissions militaires 
envers les autorités civiles est marquée par le fait que dans un tiers des cas les commissions militaires ne voulaient ni acquitter ni condamner, et renvoyèrent simplement les prévenus devant les administrateurs départementaux, ou devant le ministre de la Police générale.

À la longue, les efforts du Directoire pour limiter le champ d'action des commissions militaires aux émigrés représentant une menace pour les républicains et pour empêcher les commissions d'outrepasser leur autorité, finirent par diminuer l'impact de celles-ci. Ce déclin de l'activité des commissions militaires ne se fit pas en douceur. En fait, les modes de condamnation révèlent différentes phases. La conséquence provient autant des efforts du gouvernement pour rétablir l'ordre que de l'influence du républicanisme radical pendant les deux dernières années du Directoire.

La phase initiale s'étendit du 19 fructidor an $V$ au coup d'État antijacobin du 22 floréal an VI. La moitié de tous les cas entendus par les commissions militaires fut jugée pendant ces neuf mois. (voir graphique infra p. 43). Pendant cette période de résurgence jacobine, les activités des commissions militaires passèrent d'opérations extrêmement arbitraires à des opérations assez bien contrôlées. D'abord, la loi du 19 fructidor engendra un exode massif des émigrés rentrés, en particulier des villes fédéralistes du $\operatorname{Midi}^{57}$; des rafles de grande envergure commencèrent presque immédiatement à travers les régions où l'émigration avait été importante en 1793. Après deux mois d'arrestations, les autorités avaient réuni une centaine de personnes en prison à Strasbourg et deux cents au Fort Saint-Jean, à Marseille, toutes en attente d'être jugées par une commission militaire ${ }^{58}$. Un tel zèle était caractéristique de la résurgence jacobine et certains cas étaient de toute évidence de purs règlements de comptes. Déon Modeste, habitant de La Valette dans le Var fut poignardé neuf fois sur la route qui le menait à Toulon pour son procès, sans doute à cause de son rôle dans l'assassinat récent d'Aubert, lui aussi de La Valette, le commissaire du Directoire attaché au tribunal correctionnel de Toulon. Un des complices de Modeste, André Geoffroy dit le Chevalier, fut exécuté plus tard comme émigré, mais le gouvernement intervint rapidement pour faire transférer plusieurs autres soi-disant égorgeurs royaux devant les tribunaux criminels ${ }^{59}$.

Bien que les jacobins profitèrent de la loi du 19 fructidor an $\mathrm{V}$, les commissions militaires qu'elle créa ne devraient pas être associées exclusivement aux républicains radicaux. À un répit relatif durant les trois mois suivant les élections du printemps de l'an VI succéda une nouvelle vague de procès et d'exécutions au cours de l'été suivant. Cette recrudescence soudaine faisait partie des mesures sévères prises contre toute forme de crime violent et de résistance au régime. En messidor an VI (juin-juillet 1798), les législateurs autorisèrent le Directoire à ordonner des visites domiciliaires pendant un mois afin d'arrêter les espions anglais, les émigrés rentrés, les prêtres soumis à la déportation, les égorgeurs, les brigands et les chefs de chouans. Cela produisit un deuxième groupe d'émigrés renvoyés devant les commissions militaires.

Une dernière vague de procès, bien que plus restreinte et moins meurtrière que les deux précédentes, suivit les élections du printemps de 1799 et dura pendant «les cent jours jacobins $»^{60}$. Ainsi, contrairement aux conclusions de Victor Pierre, les commissions militaires n'avaient pas disparu après le contre-coup législatif du 30 prairial an VII (18 juin 1799) qui mit fin au soi-disant Second Directoire. En fait, quelques exécutions eurent lieu même après le 18 brumaire an VIII et les derniers procès eurent lieu huit mois plus tard, à Coblentz ${ }^{61}$, lieu hautement symbolique de 
l'émigration antirévolutionnaire. Néanmoins, le Consulat désavoua vite la politique de la terreur directoriale.

32 L'adjonction de huit cents causes judiciaires traitées par les commissions militaires, auparavant ignorées, aux deux cents relevées par Pierre, nous oblige à reconsidérer les mythes que les contemporains et les historiens ont générés sur les massacres judiciaires commis après Fructidor. Plutôt que de se laisser emporter par des considérations morales sur la cruauté et l'injustice perpétrées au nom de la République dans le style de Victor Pierre et de Ludovic Sciout, nous avons cherché à comprendre pourquoi le Directoire eut recours à une forme de violence d'État qui démentit sa prétention initiale de régime post-révolutionnaire.

Alors que pendant l'an II, la Terreur avait été menée «sur une base nationale de soustraitants $»^{62}$, la répression qui suivit Fructidor était une opération bien gouvernementale. La lecture attentive des archives permet de constater l'effort soutenu du gouvernement pour réduire systématiquement le champ d'action des commissions, non pas afin de les neutraliser, mais pour en faire des instruments raffinés de la répression, dirigés avec le maximum de précision contre les opposants considérés comme les plus implacables du régime. Les opérations des commissions militaires révèlent un rythme dans les vagues de répression. La première vague fut médiocrement contrôlée, alors que les suivantes furent à la fois moins intenses mais plus concentrées. Ceci fut le fruit d'une politique délibérée. Bien que l'habitude révolutionnaire de s'en prendre aux nobles et aux prêtres ne disparût pas, les preuves nouvelles concernant les victimes des commissions militaires mettent à jour les intentions du gouvernement de ne juger et de n'exécuter que les notables émigrés, considérés comme les véritables ennemis de la République. Pour comprendre cette action, il faut remettre les commissions militaires, l'instrument par excellence de la "terreur directoriale », dans le contexte d'une illégalité endémique et d'une violente politique générale. C'est dans ce contexte seulement qu'on peut apprécier jusqu'à quel point la persécution des émigrés rentrés faisait partie d'un système de répression établi pour réprimer à la fois les désordres criminels et politiques. La résurrection des commissions militaires fut une réponse à l'effondrement presque total de l'autorité gouvernementale pendant l'été de 1797. 
Commissions militaires

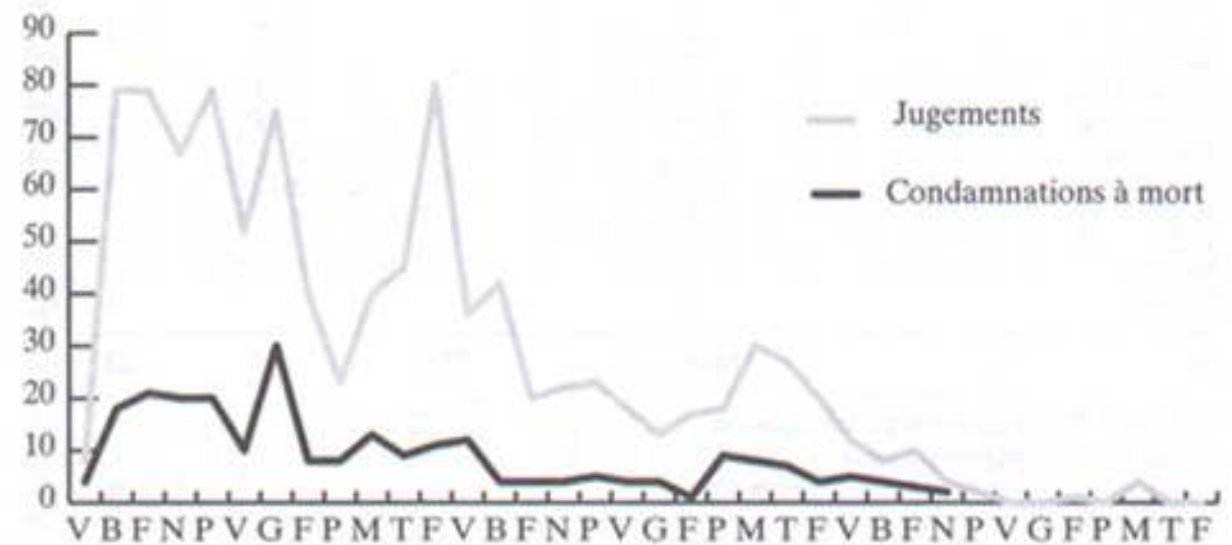

Lieux des commissions militaires et leurs sentences de conviction

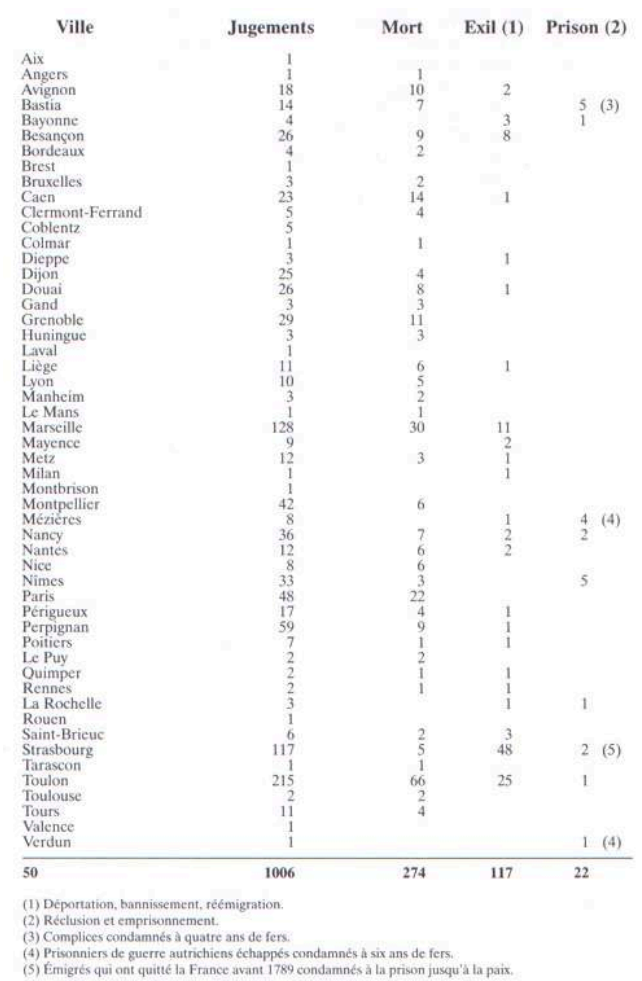




\section{NOTES}

1.Jean-Pierre GALLoIS, Dix-huit fructidor; ses causes et ses effets, Hambourg, 1799, pp. 151-152.

2.Pour un récit complet du coup d'État, ainsi qu'un aperçu général du débat sur son rapport à un complot anglo-royaliste, voir Albert MEYNIER, Le dix-huit fructidor an $V$, Paris, 1927, pp.141-216, et Georges LEFEBVRE, La France sous le Directoire, 1795-1799, deuxième édition présentée par Jean-RenésURATTEAU, Paris, 1984, pp. 418-433, pp. 729-736.

3.Pour une description détaillée de la façon dont les commissions militaires devaient en principe fonctionner, voir Service historique de l'Armée de Terre (ci-après S.H.A.T.) J2 51, ministre de la Justice au ministre de la Guerre, 21 pluviôse an VI. La convocation des commissions se faisait provisoirement et les juges devaient changer pour les différents cas; néanmoins, nombreuses étaient celles qui devinrent quasi permanentes comme le ministre de la Guerre le reconnut dans une circulaire: S.H.A.T. B13, 12 ventôse anVI. 4.Jean-Pierre MAYER (éd.), CEuvres, papiers et correspondance d'Alexis de Tocqueville, Paris, 1953, vol. 2, partie 2, p. 270.

5.Georges LEFEBVRE, La France sous le Directoire..., op. cit., emploie l'expression « terreur directoriale "; Denis WORONOFF, La République bourgeoise de Thermidor à Brumaire, 1794-1799, Paris,1972, p.195, préfère l'expression « terreur fructidorienne »; François FURET et Denis RICHET, La Révolution française, Paris, 1965, p. 488, utilisent les deux expressions.

6.La Terreur sous le Directoire: Histoire de la persécution politique et religieuse après le coup d'État du 18 fructidor, Paris, 1887; 18 Fructidor: Documents pour la plupart inédits recueillis et publiés pour la Société d'histoire contemporaine, Paris, 1893; La déportation ecclésiastique sous le Directoire: Documents inédits recueillis et publiés pour la Société d'histoire contemporaine, Paris, 1896. La position anti-révolutionnaire de ces œuvres était directement représentée dans le réquisitoire monumental de Ludovic Sciout, Le Directoire, 4 vol., Paris, 1895-1897.

7.Plus d'un dixième des jugements cités dans 18 Fructidor manque de noms ou de dates précises. De plus, il n'y a que 87 noms de familles pour 158 victimes supposées (55\%) dans son appendice III: « Liste, par ordre chronologique, des condamnations à mort prononcées par les diverses commissions militaires» (pp. 662-664). Victor Pierre indique dans ses notes sur les documents publiés que trois des condamnations à mort furent prononcées par d'autres tribunaux que les commissions militaires et qu'une autre victime était probablement un duplicata. Néanmoins, il inclut tous les quatre dans sa liste sommaire sans fournir la moindre explication (18 Fructidor..., op. cit., pp. 349, 380, 416, 449). De plus, il ignorait que Blayal (de Grans) et Ferrand (d'Aix) n'étaient pas condamnés par la commission militaire siégeant à Marseille, mais par le premier 
conseil de guerre de la huitième division militaire (Archives départementales des Bouches-du-Rhône 2R 465, 466).

8.Par exemple, Jean JAURÈs, Histoire socialiste de la Révolution française, tome 5: Thermidor et Directoire par Gabriel DEVILLE, Paris, 1904, p. 424; Georges LEFEBVRE, La Révolution française, Paris, 1reéd., 1930, 6e éd., 1968, p. 500; François FURET, La Révolution: de Turgot à Jules Ferry, 1770-1880, Paris, 1988, p.199, and William DOYLE, The Oxford History of the French Revolution, Oxford, 1989, p.334. Évidemment, ces chiffres se trouvent également dans les ouvrages de référence: par exemple, Jacques GODECHOT, Les Institutions de la France sous la Révolution et l'Empire, 3e éd., Paris,1985, p. 479, et Colin JonEs, The Longman Companion to the French Révolution, London, 1988, p. 51.

9.18 Fructidor..., op. cit., p. xxxIv. Ceci est incohérent en soi: 54 des 108 victimes non ecclésiastiques mentionnées ne possèdent pas plus d'identitié pour Victor Pierre que les cadavres anonymes à Toulon, mais ceci peut uniquement être discerné en étudiant sa documentation.

10.Les condamnations signifiaient la mort, la déportation, la ré-émigration, ou la réclusion. Une douzaine de jugements imposèrent illégalement des peines de prison à des personnes qui n'étaient pas justiciables des commissions militaires créées par la loi du 19 fructidor. Voir note 53.

11.Trente-neuf jugements concernant ceux qui se déroulèrent à Paris se trouvent dans les cartons du S.H.A.T. C183, 4, 5; ceux qui proviennent d'ailleurs en France sont éparpillés parmi les 489 cartons de la série S.H.A.T. J2 méconnus jusqu'en 1993. Je tiens à remercier Monsieur Frankhauser du Service historique de l'Armée de Terre pour m'avoir aidé à trouver cette série et pour avoir fait préparer par la suite un répertoire numérique. Victor Pierre ignorait complètement l'existence de 650 individus cités dans ces jugements seuls. Une grande quantité d'autres jugements ajoutent des informations fondamentales dont il ne disposait pas. Charles J. Doyle, « The Judicial Reaction in South-eastern France, 1794-1800 ", thèse de doctorat dactylographiée, Université d'Oxford, 1987, m'amena aux Archives nationales (ci-après A.N.) BB18880, qui contiennent des informations sur 161 personnes jugées à Toulon (dont 138 n'apparaissent pas dans les séries J2). D'autres cas isolés ou d'autres informations complémentaires viennent des séries S.H.A.T. B13, A.N. F7, Archives départementales de la Gironde et des Bouches-du-Rhône séries 2R, et des histoires locales. Dans toute cette documentation, le plus grand nombre évident de lacunes vient des procès tenus à Strasbourg: il y existe 117 jugements, mais seulement l'un d'entre eux est de la période entre le 1ervendémiaire et le 1er thermidor an VII. Les extrapolations tirées du nombre de procès qui eurent lieu ailleurs pendant ces dix mois et le nombre de procès qui eurent lieu à Strasbourg avant et après cette période, indiquent l'absence de 35 à 45 jugements. Cependant, étant donné le bas pourcentage d'exécutions repérées, situer ces jugements n'ajouterait probablement que deux ou trois peines de mort au total. 12.La divergence tient au fait que certains individus furent soumis à deux ou trois procès. Par exemple, Charles Desroches-Chassay fut arrêté au Château de Maranda (Charente) et passa en jugement devant une commission militaire à Poitiers. Le 28 pluviôse an VI, celle-ci conclut au transfert du prévenu devant ses « juges naturels » dans la Haute-Vienne où il était né. Cependant, une commission militaire de Périgueux conclut, le 16 ventôse an VI qu'il n'était pas le Desroches-Chassaix figurant sur la liste nationale des émigrés et le libéra. Le département de la Haute-Vienne décria ce jugement comme étant le résultat de faux documents et le gouvernement le fit arrêter 
de nouveau à Paris, où une troisième commission militaire le condamna à mort le 18 messidor an VI: S.H.A.T. J2 285, J2 283, c18 5.

13.Il ne serait pas nécessaire d'insister sur ce point si ce n'était pour l'importance des ouvrages tels que ceux de Isser woloch, The Jacobin Legocy: The Democratic Movement Under the Directory, Princeton, 1970, qui néglige le rôle joué par le soi-disant néojacobinisme dans le façonnage de solutions révolutionnaires en réponse aux problèmes d'ordre et de stabilité sous le Directoire. Une rectification partielle peut être trouvée dans l'ouvrage de Christine PEYRARD, Les Jacobins de l'Ouest, Paris, 1996, pp.307-362. Les difficultés rencontrées pour maintenir une sociabilité démocratique et pour produire des solutions républicaines flexibles durant la douloureuse période du Directoire font l'objet de plusieurs ouvrages récents, plus particulièrement le numéro spécial des Annales historiques de la Révolution française (1994, n²) présenté par Bernard GAINOT et de nombreux articles parus dans Philippe BOURDIN et Bernard GAINOT (dir.), La République directoriale, 2 vol., Clermont-Ferrand et Paris, 1998.

14.Voir Roger DUPUY et Marcel MORABITO (dirs.) 1795: Pour une République sans Révolution, Rennes, 1996, et Michel VovelLE, (dir.) Le tournant de l 'an III: Réaction et Terreur blanche dans la France révolutionnaire, Paris, 1997.

15.La loi du 14 frimaire anV annula l'article 10 de la loi du 3 brumaire anIV en redonnant de la force aux lois anticléricales de 1792-1793, mais de ce fait laissa dans le vague le statut des prêtres réfractaires jusqu'à ce que la loi du 7 fructidor anV abroge l'ancienne législation. Entre-temps, la loi du 9 messidor anV abolit la loi du 3 brumaire an IV, permettant aux vendémiairistes et aux parents d'émigrés d'exercer des fonctions publiques.

16.J.ADHER, « La conspiration royaliste dans la Haute-Garonne en l'anIV et l'anV », Révolution française XII (1901); Philippe BOURDIN, « Les « Jacobins » du bois de Cros (Clermont-Ferrand, anV): chronique d'un massacre annoncé ", Annales historiques de la Révolution française, 1997, pp. 249-304; sur les troubles autour de Castres, voir A.N. BB18 684 et F7 7296.

17.François de Neufchâteau fut particulièrement éloquent sur la montée du crime et sur le déclin du zèle administratif à partir des élections de l'anV. Voir « Ministre de l'Intérieur aux administrations centrales et aux administrations municipales, 15 fructidor an V » dans Randonneau, Recueil des lettres, circulaires, instructions, programmes, discours, et autres actes publics, émanés du Citoyen François (de Neufchâteau), pendant ses deux exercices du ministère de l'Intérieur, 2 volumes (Paris, an VII), i. liv-lix.

18.Voir la collection de ses écrits pendant le Directoire éditée par Philippe RAYNAUD, Benjamin Constant, Paris, 1988, en particulier Des effets de la terreur rédigé en mai 1797. 19.Ceci est très évident dans les discours de soutien à l'exclusion des anciens nobles des droits de citoyenneté après le coup d'État. Voir: Opinions de Rousseau sur la résolution relative aux ci-devant nobles et anoblis, Conseil des Anciens, séance du 7 frimaire an VI; Motion d'ordre de Gay-Vernon, sur la discussion relative aux ci-devant nobles, Conseil des Cinq-Cents, séance du 25 vendémaire an VI; Opinion de P.Guchan, sur la résolution relative aux ci-devant nobles et anoblis, Conseil des Anciens, séance du 9 frimaire an VI.

20.S.H.A.T. J2 212, Platel, 15 pluviôse anVI; S.H.A.T. J2 126, Estrangin, 2 frimaire anVI, et Donnadieu, 9 germinal an VI; S.H.A.T. J2 16, Cosnac, 13 germinal an VI.

21.Donald GREER, The Incidence of the Emigration During the French Révolution, Gloucester, Mass., 1966, estime un nombre d'environ 130000 émigrés.

22.La loi du 26 août 1792 avait exigé le départ de France de tous les prêtres réfractaires sous risque d'être déportés en Guyane. Bien qu'ils fussent toujours qualifiés de 
" déportés ", même s'ils ne faisaient que se cacher, ils étaient réellement des exilés, la plupart ayant été bannis, non pas déportés. Uniquement ceux de plus de soixante ans ou infirmes furent exemptés et placés sous surveillance. La loi du 13 septembre 1793 les assimila aux émigrés, ce qui signifiait, selon la loi contre les émigrés du 25 brumaire an III, un accroissement des sanctions à leur égard lesquelles pouvaient aller de la déportation au passage devant un peloton d'exécution.

23.Circulaires du ministre de la Police générale, 3 et 14 brumaire an VI. Pour celles-ci et d'autres circulaires mentionnées ci-dessous, voir S.H.A.T. C18 4, généralités.

24.Jean GIRARDOT, Le département de la Haute-Saône pendant la Révolution, 3 vol., Vesoul, 1973, III, 314-316; S.H.A.T. J2 144.

25.C'est-à-dire, ceux qui étaient couverts par les lois du 22 nivôse et le 4e jour complémentaire anIII et qui obéissaient à leurs conditions. La décision de les exempter de la loi du 19 fructidor anV date du 12 nivôse anVI.

26.La différence reposait sur les arguments émis plus tôt par Benjamin CONSTANT, Les réflexions de Marchéna sur les fugitifs inséré dans Le Républicain français; Pierre-Louis, comte de ROEDERER, Des fugitifs français et des émigrés (Paris, 28 thermidor an III, 1795) et Jean-Jacques LeULIETTE, Des émigrés français, ou réponse à M. De Lally-Tollendal (Paris, 1797). La distinction persista pendant les débats sur la résolution adoptée par le Conseil des Cinq-Cents, le 17 messidor an V, mais échoua finalement au Conseil des Anciens, le 12 fructidor an V. Voir en particulier le rapport présenté par Harmand au Conseil des Anciens le 7 fructidor an $\mathrm{V}$ et reproduit dans Le Moniteur (réimpression), XXVIII,784. 27. Circulaire du ministre de la Police générale, 18 brumaire an VII.

28. Rapport de commission au Conseil des Cinq-Cents le 3 vendémiaire an $\mathrm{V}$ dans $L e$ Moniteur universel, an V, p. 26.

29.A.N. BB18 880, (Toulon) 28 nivôse an VI, Thérèse Mayan, et 13 pluviôse an VI, Claire Macadre, veuve Provençal; S.H.A.T. J2 126, (Marseille) 11 germinal an VI, Claire Verdier, veuve Rey; (Toulon) 19 germinal an VI, Christine Aiguier, épouse de Polycarpecommuée. Aiguier fut jugée avec Claire-Élizabeth Fournier, qui aurait aussi pu être condamnée à mort, mais elle avait eu un accouchement difficile, le 18 fructidor an $\mathrm{V}$ et ne s'en remit pas à temps pour obéir à la loi du 19 fructidor leur exigeant de quitter la République dans les deux semaines et fut, ainsi, condamnée à la réémigration. Le ministre de la Police générale émit une circulaire le 2 floréal an VI.

30.Un total de 67 femmes furent jugées (soit $0,7 \%$ du total): en plus des 5 condamnées à mort, 14 furent condamnées à la réémigration, 43 furent libérées et 5 furent envoyées devant les autorités civiles (l'équivalence pratique de l'exemption).

31.La circulaire du ministre de la Police générale datant du 6 thermidor an VI autorisa la surveillance admninistrative plutôt que les poursuites judiciaires pour ceux que le statut professionnel aurait qualifié pour l'exemption couverte par la loi du 22 nivôse et $\mathrm{du} 4 \mathrm{e}$ jour complémentaire anIII. Comme les administrateurs du Bas-Rhin le présentent dans leur lettre au ministre de la Police générale le 23 pluviôse an VIII, « le Directoire exécutif ayant d'ailleurs manifesté par la suite des dispositions plus douces à leur égard, la Commission militaire a cru devoir entrer dans ces vues, en ne suivant pas à la lettre les rigueurs de la loi ». Cité par Rodolph Reuss, La grande fuite de décemhre 1793 et la situation politique et religieuse du Bas-Rhin de 1794 à 1799, Strasbourg, 1924, p. 303.

32. Circulaires du ministre de la Police générale du 12 nivôse et du 6 thermidor an VI. 33.Rapport de Tronson-Ducoudray, au sujet de la résolution concernant les fugitifs de Toulon, Conseil des Anciens, séance du 23 thermidor an V. 
34.Gordon WRIGHT, Between the Guillotine and Liberty: Two Centuries of the Crime Problem in France, Oxford, 1983, p. 34.

35.Pour les autres méthodes de répression, voir Howard G. BROWN, « From Organic Society to Security State: The War on Brigandage in France, 1797-1802 », Journal of Modern History 69, 1997, pp.661-695.

36.S.H.A.T. J2 51, 12 nivôse an VI.

37.S.H.A.T. J2 132, 3 vendémiaire an VII. Voir aussi, Howard G. BROWN, « An Unmasked Man in a Milieu de Mémoire: the Abbé Solier as Sans-Peur the Brigand-Priest ", Historical Reflections / Réflexions historiques 26 (2000): 1-30.

38.S.H.A.T. J2 289, 3 germinal an VI; PIERRE, Terreur..., op. cit., pp. 441-442.

39.Le Moniteur universel, an VI, p. 129 (29 vendémiaire an VI).

40. , an VI, p. 296 (26 brumaire an VI).

41. Ceci est central à son livre, Class, Ideology and the Rights of Nobles in the French Révolution, Oxford, 1981, où il chercha à exposer la nature irréconciliable de l'individualisme universel chez les révolutionnaires et leurs intérêts de membres de la classe bourgeoise. Puisque le régime directorial était tout aussi bourgeois que républicain, l'attaque motivée idéologiquement contre les nobles à titre d'anciens privilégiés entra en conflit avec le besoin de créer une alliance parmi les propriétaires parce que les anciens nobles constituaient une portion importante de la classe des propriétaires en France. Ceci, conclut Higonnet, mena à un cul-de-sac politique et entraîna la fin du Directoire et avec elle l'effondrement des espoirs démocratiques de la Révolution. C'est une analyse audacieuse, mais fortement sur-déterminée et qui dépend d'une interprétation de la terreur directoriale ce qui fausse sérieusement les preuves. 42.En termes numériques, les commissions militaires condamnèrent à mort 275 individus, dont 270 peuvent être identifiés par nom et par métier, profession ou statut social. Il y avait 42 nobles, 48 ecclésiastiques, 45 autres notables, 50 artisans et commerçants, 32 paysans et 9 militaires. Les informations sont insuffisantes concernant les 51 individus restants, mais il semble que seulement quelques-uns d'entre eux étaient des nobles puisqu'un statut social important était généralement inscrit pour ajouter une légitimité aux exécutions.

43.S.H.A.T. J2 126, 21 germinal an VI (Macagno), 1 fructidor an VI (Truchi), 5 vendémiaire anVII (Barmando).

44.S.H.A.T. J2 112, (Grenoble) 13 nivôse an VI, et Pierre, 18 fructidor, p. 338; S.H.A.T. C185, (Paris) 15 brumaire an VII, et Félix SCHAEDELIN, L'émigration révolutionnaire du HautRhin, Colmar, 1937, p. 122; S.H.A.T. J2 285, (Poitiers) 6 pluviôse an VI.

45.S.H.A.T. C18 5, (Paris) 19 thermidor an VI; voir aussi, Alphonse de BEAUCHAMP, Mémoires du comte Fortuné Guyon de Rochecolte, Paris, 1818, p. 217.

46. Charles JOLIVET, Les chouans du Vivarais. Essai sur l'agitation contre-révolutionnaire dans l'Ardèche sous le Directoire, Lyon, 1930, pp. 29, 40-41, 59-64; Ernest DAUDET, Les conspiration de Pichegru et les complots royalistes du Midi et de l'Est, 1795-1797, Paris, 1901, pp. 150-152, 294-316.

47.Jean VIDALENC, Les émigrés français, 1789-1815, Paris, 1963, pp. 123-124.

48.Elles sont responsables de 343 jugements, soit un tiers du total.

49. Ces exemples proviennent du S.H.A.T. J2 132, (Montpellier) 6 ventôse an VI, Escoffier, 29prairial an VII, Mistral; S.H.A.T. J2 126, (Toulon) 6 frimaire an VI, Lion, 9 vendémiaire an VII, Fougue, membre du tribunal martial; (Marseille) 6 germinal an VI, Delille, 11 germinal an VI, Morin, 12germinal an VI, Étienne, gendarme; A.N. BB18 880 
(Toulon), 11 brumaire an VI, Geoffroy; 7 frimaire an VI, Marquisant, gendarme; 18 frimaire an VI, Vidal, huissier; 19 nivôse an VI, Calze, gendarme.

50.S.H.A.T. C18 5, (Paris) 15 thermidor an VI; S.H.A.T. J2 200, (Caen) 27 prairial an VII.

$\mathbf{5 1 . 8 5 5 3}$ autres prêtres (y compris 7847 de Belgique) furent condamnés à la déportation, mais ne furent pas réellement envoyés en Guyane, ni aux îles de Ré ou d'Oléron, car la vaste majorité d'entre eux ne fut jamais appréhendée, et ceux qui le furent restèrent simplement en prison en France. Le nombre total de morts comprend ceux qui moururent à Rochefort et à bord de navires: PIERRE, Terreur..., op. cit., pp. 423-461. 52.Alexandre Friquet, marchand épicier, (Douai, 6 frimaire an VI) et André Le Pape, vicaire de Rosquelven, Côtes-du-Nord, (Saint-Brieuc, 1 nivose an VI) moururent en Guyane, tandis qu'Antoine-Léopold Buchet, curé de Breury-lès-Faverney, Haute-Saône, (Besançon, 7 pluviôse an VI) mourut en route sur le Bayonnaise. En outre, Jean-François Viennot, curé d'Autet, Haute-Saône, (Besançon, 7pluviôse an VI), mourut à Dijon, âgé de 74 ans, en route pour Rochefort: S.H.A.T. J2 212; GIRARDOT, Haute-Saône..., op. cit., III, 314; PIERRE, 18 fructidor..., op. cit., pp. 260-261; PIERRE, Terreur..., op. cit., pp. 420-421, 425, 431.

53.S.H.A.T. J2 81, (Strasbourg); J2 126, (Marseille); J2 51 (Marseille) mal classé; A.N. BB18 880 (Toulon).

54.S.H.A.T. J2 152, (Saint-Brieuc) 6 frimaire an VI et 7 pluviôse an VI.

55.S.H.A.T. J2 291, (Bastia) 8 brumaire an VII; J2 16, (Mézières) 3 frimaire an VIII, (Verdun) 15frimaire an VIII; S.H.A.T. J2 81, (Strasbourg) 12 floréal an VI et 29 messidor an VI; PIERRE, 18 fructidor..., op. cit., pp. 420-422.

56.A.N. F7 4374. Voir également A.N. BB18 271, Général de division Montigny, commandant la 6edivision militaire, à l'administration centrale du Doubs, 5e jour complémentaire an VI, 8 messidor anVII, et 10 prairial an VIII.

57.Bien qu'il s'agisse sans aucun doute d'une exagération, la nouvelle municipalité de Marseille affirma que 15000 personnes fuirent la ville après que la loi y fut promulguée: Antoine BERNARD, « Le 18fructidor à Marseille et dans les Bouches-du-Rhône », La Révolution française, XLI (juillet-septembre 1901), pp. 200-201.

58.S.H.A.T. J2 81, adjutant-général Croz, président de la commission militaire, au ministre de la Guerre, 8 frimaire an VII; Sciout, Directoire..., op. cit., iii, 32.

59.A.N. BBl8 880, 4 et 7 brumaire, et 22 pluviôse an VI; Journal de Toulouse, ou l'Antiroyaliste, 26 brumaire VI. Pour une description détaillée du rôle joué par un émigré dans la longue réaction thermidorienne en Provence, voir le cas de Claude Ferrand, condamné à mort à Montpellier, le 23 vendémiaire anVIII (ADG 2R 1165).

60.Jean-Paul BERTAUD, Bonaparte prend le pouvoir, Bruxelles, 1987, p. 109.

61.Georges LEFEBVRE, Révolution française..., op. cit., p. 500, déclare qu'une seule exécution eut lieu après le mois de mars 1799, quand en fait, le nombre est au moins de 45: les deux dernières exécutions eurent lieu le lendemain de Noël 1799. Voir S.H.A.T. J2 126, (Toulon) 5 nivôse an VIII. Pour les derniers jugements, voir S.H.A.T. J2 314, 28 floréal et 15 messidor an VIII.

62.Richard Совв, The Police and the People: French Popular Protest, 1789-1820, Oxford, 1970 , p.189. 


\section{RÉSUMÉS}

Sur la base d'archives inédites du Service historique de l'Armée de Terre, l'auteur étudie la répression judiciaire qui a suivi le coup d'État du 18 fructidor anV. Ses sources mettent à jour notamment le rôle des commissions militaires, le sort réservé à plus de mille individus passés en jugement, les rythmes de cette répression, enfin la composition sociologique du groupe étudié. H.Brown montre non seulement comment le Directoire a eu pour objectif tout à la fois de maintenir l'ordre public et de préserver une certaine stabilité politique, mais aussi comment il a porté ses coups les plus sévères contre tous ceux qu'il estimait être ses réels adversaires politiques et non pas contre les seuls émigrés ou les prêtres réfractaires.

Myths and Massacres: Reconsidering the Directoire Terror. Using unpublished source material from the French Army Historical Service, the author reviews the judicial repression which followed the coup of 18 Fructidor YearV. The sources reveal the role played by the military commissions, the lot of more than a thousand individuals indicted, the rhythm of repression and also the sociological make-up of the group surveyed. He shows not only how the Directoire aimed at preserving public order together with a certain political stability, but how it clamped down hardest on those it considered its real political adversaries, not just the emigrés or refractory priests.

INDEX

Mots-clés : Directoire, Fructidor, Terreur directoriale, commissions militaires, justice

\section{AUTEUR}

HOWARD G. BROWN

Department of History, State University of New York at Binghamton P.O Box 6000, Binghamton, NY 13902-6000, États-Unis 